\title{
$\mathrm{In}^{3+}$ 共掺杂诱导 $\mathrm{NaY}_{0.95-x} \mathrm{Yb}_{0.03} \mathrm{Er}_{0.02} \mathrm{~F}_{4}$ 相转变与上转换发光增强研究

\author{
俞瀚*, $a$ 黄清明 $a, b$ 曹文兵 ${ }^{a}$ 张新奇 ${ }^{b}$ 俞建长 ${ }^{a}$ \\ ( ${ }^{a}$ 福州大学材料科学与工程学院 福州 350108) \\ ${ }^{b}$ 福州大学测试中心 福州 350002)
}

\begin{abstract}
摘要 引入 $\mathrm{In}^{3+}$ 作为新的掺杂离子通过水热法合成了 $\mathrm{In}^{3+}$ 与 $\mathrm{Er}^{3+}, \mathrm{Yb}^{3+}$ 共掺杂的 $\mathrm{NaYF}_{4}$ 上转换发光材料. 通过 XRD 表 征及 Rietveld 精修, SEM 以及 TEM 研究了 In 掺杂引起的 $\mathrm{NaYF}_{4}$ 形貌与晶体结构的变化. 通过发射光谱与发光衰减曲 线表征了掺杂 $\mathrm{NaYF}_{4}$ 的上转换发光性能，以研究晶体微观结构与上转换发光性能之间的关系. 结果表明随着 In 掺杂量 的增加, 立方相 $\mathrm{NaYF}_{4}$ 的晶格畸变不断增强且发生由立方相向六方相的转变. 对于六方相 $\mathrm{NaYF}_{4}$, 其上转换发光强度 随着 In 含量的提高不断增强并当 In 含量为 $3 \%$ 时达到最大值. 该研究结果可以有助于设计与合成具有优良上转换发光 性能的材料.
\end{abstract}

关键词 上转换; $\mathrm{In}^{3+}$ 掺杂; 相转变; 发光; 氟钎酸钠

\section{Phase Transition Induction and Upconversion Luminescence Enhancement of $\mathrm{NaY}_{0.95-x} \mathrm{Yb}_{0.03} \mathrm{Er}_{0.02} \mathrm{~F}_{4}$ by $\mathrm{In}^{3+}$ Codoping

\author{
$\mathrm{Yu}, \mathrm{Han}^{*, a} \quad$ Huang, Qingming ${ }^{a, b} \quad$ Cao, Wenbing $^{a} \quad$ Zhang, Xinqi $^{b} \quad$ Yu, Jianchang $^{a}$ \\ $\left({ }^{a}\right.$ College of Materials Science and Engineering, Fuzhou University, Fuzhou 350108) \\ ( ${ }^{b}$ Instrumental Analysis \& Measurement Center, Fuzhou University, Fuzhou 350002)
}

\begin{abstract}
In}^{3+}$ was taken as a new dopant to codope $\mathrm{NaYF}_{4}$ with $\mathrm{Er}^{3+}$ and $\mathrm{Yb}^{3+}$ by hydrothermal synthesis in this manuscript. How to improve phase transition of $\mathrm{NaYF}_{4}$ from cubic to hexagonal and how to improve upconversion luminescence performance of hexagonal $\mathrm{NaYF}_{4}$ are both important problems because hexagonal $\mathrm{NaYF}_{4}$ is the most efficient matrix material for upconversion. Considering these two problems, we report the study of crystal structure and upconversion emission of $\mathrm{In}^{3+}$-codoped $\mathrm{NaYF}_{4}$ to discover phase transition mechanism and upconversion luminescence properties changed with different $\mathrm{In}^{3+}$ codoping concentration. $\mathrm{NaY}_{0.92} \mathrm{Yb}_{0.03} \mathrm{Er}_{0.02} \mathrm{~F}_{4}$ was tridoped with $0,2,4,6,8$ and $10 \mathrm{~mol} \% \mathrm{In}^{3+}$ at $170{ }^{\circ} \mathrm{C}$ for $8 \mathrm{~h}$ in order to study phase transition of $\mathrm{NaYF}_{4}$. The other group of samples was prepared by the same procedure except $\mathrm{In}^{3+}$ codoping concentration was replaced as $0,1,2,3,4$ and $5 \mathrm{~mol} \%$ and the reaction proceeded at $190{ }^{\circ} \mathrm{C}$ for $12 \mathrm{~h}$ in order to obtain pure hexagonal $\mathrm{NaYF}_{4}$ for study of its upconversion luminescence. The change of morphologies and crystal structural transition caused by $\mathrm{In}^{3+}$ codoping was explored by XRD, Rietveld refinement, SEM and TEM analysis methods, while the upconversion luminescence was characterized by emission spectra and luminescence decay curves, for the relationship between crystal microstructure and luminescence properties to be discussed in our investigation. On the basis of these characterizations we found that with rising of the $\mathrm{In}^{3+}$ codoping concentration, the phase transition of $\mathrm{NaYF}_{4}$ from cubic to hexagonal was improved. The intensity of upconversion luminescence was also found to be increased to the maximum when 3 mol\% $\mathrm{In}^{3+}$ was codoped into hexagonal $\mathrm{NaYF}_{4}$. The analysis and discussion discovered that the phase transition of $\mathrm{NaYF}_{4}$ was correlated with the lattice distortion of cubic $\mathrm{NaYF}_{4}$ improved by $\mathrm{In}^{3+}$ codoping. The intensity of upconversion luminescence was proved to be increased by the asymmetry of the local crystal field changed with the $\mathrm{In}^{3+}$ codoping concentration. This investigation may be helpful for design and synthesis of other functional materials.

Keywords upconversion; $\mathrm{In}^{3+}$ codoping; phase transition; luminescence; $\mathrm{NaYF}_{4}$
\end{abstract}

\section{1 引言}

近年来, 将长波激发光转换为短波发射的上转换发 光正得到研究者们的大量关注 ${ }^{[1 \sim 3]}$. 上转换发光材料在 包括生物标识及成像 ${ }^{[4 \sim 8]}$ 、固体激光器 ${ }^{[9]}$ 、温度传感
器 ${ }^{[10,11]}$ 、催化 ${ }^{[12]}$ 以及能量转化与利用 ${ }^{[13 \sim 16]}$ 等方面表现出 广泛的应用前景. 在上转换发光材料中, 六方相的 $\mathrm{NaYF}_{4}$ 由于其低声子能量的特点作为效率最高的上转 换发光材料尤其引人注目 ${ }^{[17]}$. 清华大学李亚栋院士课 题组与北京大学严纯华院士课题组在国际上率先开展

\footnotetext{
*E-mail: fjfzyh@fzu.edu.cn

Received July 27, 2013; published October 12, 2013.
}

Supporting information for this article is available free of charge via the Internet at http://sioc-journal.cn.

Project supported by the Fujian Province Natural Science Fund (No. 2013J05027) and the Fujian Province Education-Science Project for Middle-aged and Young Teachers (No. JA13050)

项目受福建省自然科学基金(No. 2013J05027)与福建省中青年教师教育科研项目(No. JA13050)资助. 
了包括 $\mathrm{NaYF}_{4}$ 在内的稀土化合物纳米材料合成及其光 学性质研究 ${ }^{[18 ~ 21]}$. 许多研究工作都致力于改善六方相 $\mathrm{NaYF}_{4}$ 的上转换发光性能, 如形貌与尺寸控制 ${ }^{[22 ~ 24]}$, 表 面化学修饰 ${ }^{[25]}$, 壳核结构的合成 ${ }^{[14,15]}$ 与多稀土离子共掺 杂 ${ }^{[26]}$. 然而由于上转换发光机制的复杂性 ${ }^{[27]}$, 在提高六 方相 $\mathrm{NaYF}_{4}$ 上转换发光性能方面还有许多探索要做. 同 时, 由于六方相 $\mathrm{NaYF}_{4}$ 的声子能量明显低于立方相 $\mathrm{NaYF}_{4}$, 如何在温和的条件下, 比如较低的温度下或者 较短的反应时间里促使立方相 $\mathrm{NaYF}_{4}$ 向六方相转变也 是一个重要的课题.

众所周知, 掺杂是一种引入或改善材料性能的手 段, 广泛应用于功能材料的合成中 ${ }^{[28,29]}$. 一方面, 根据 Judd-Ofelt 理论 ${ }^{[30,31]}$, 稀土离子的上转换发光性能受到 其周围局部晶体场的影响效应明显, 而晶场能够为掺杂 离子所改变. 另一方面, 离子掺杂可以改变相转变的机 制 $^{[32]}$, 这使得引入半径较小的掺杂离子常常会导致低 密度相向高密度相的相转变温度的降低. 本文中, 考虑 到 $\mathrm{In}^{3+}$ 离子半径 $(80 \mathrm{pm})$ 比 $\mathrm{Y}^{3+}$ 离子半径 $(90 \mathrm{pm})$ 小, 我们 引入 $\mathrm{In}^{3+}$ 作为掺杂离子对 $\mathrm{NaY}_{0.95-x} \mathrm{Yb}_{0.03} \mathrm{Er}_{0.02} \mathrm{~F}_{4}$ 进行共 掺杂, 以起到诱导 $\mathrm{NaYF}_{4}$ 在更低温度下发生相转变同时 调节局部晶体场的对称性以改善上转换发光性能两方 面作用. 而且, 通过对 $\mathrm{In}^{3+}$ 共掺杂 $\mathrm{NaYF}_{4}$ 的晶体结构与 上转换发光性能的研究有助于揭示 $\operatorname{In}^{3+}$ 共掺杂诱导相转 变与发光性能变化的机制, 为上转换发光功能材料的设 计与合成提供帮助.

\section{2 结果与讨论}

\section{$2.1 \mathrm{In}^{3+}$ 共掺杂诱导相转变}

$\mathrm{NaY}_{0.92} \mathrm{Yb}_{0.03} \mathrm{Er}_{0.02} \mathrm{~F}_{4}$ 与 $0,2,4,6,8,10 \mathrm{~mol} \% \mathrm{In}^{3+}$ 共 掺杂, 在 $170{ }^{\circ} \mathrm{C}$ 烘箱中恒温反应 $8 \mathrm{~h}$ 得到的样品分别标 记为 $\mathrm{A} 0, \mathrm{~A} 1, \mathrm{~A} 2, \mathrm{~A} 3, \mathrm{~A} 4, \mathrm{~A} 5$, 用于相转变的研究. 样品 $\mathrm{A} 0 \sim \mathrm{A} 5$ 三价阳离子的元素定量分析如支持信息表 1 所 示, 元素分析结果与实验设计基本相符. $\mathrm{X}$ 射线衍射图 谱(XRD)如图 1 所示, 所有的衍射谱都能够标识为立方 相 $\mathrm{NaYF}_{4}\left(\mathrm{JCPDS}\right.$ 卡片号 77-2042) 与六方相 $\mathrm{NaYF}_{4}$ (JCPDS 卡片号 28-1192)的混合物, 且没有其它未标明 的衍射峰, 表明每个样品都由两相组成. 所有的衍射谱 都使用 fullprof 软件, 以六方相 $\mathrm{NaYF}_{4}(\mathrm{ICSD} 79157, a=$ $0.596 \mathrm{~nm}, c=0.353 \mathrm{~nm}$, 空间群 $P-3$ )与立方相 $\mathrm{NaYF}_{4}$ (ICSD 060257, $a=0.547 \mathrm{~nm}$, 空间群 $F m-3 m$ ) 的晶体结构 作为初始结构模型进行 Rietveld 精修. 所有精修结果的 各评价 $R$ 因子都小于 6 , 计算图谱与实测图谱吻合的很 好, 如图 1 所示. 结构精修得到两相含量的定量结果与 立方相 $\mathrm{NaYF}_{4}$ 的晶胞参数表示在图 2 中. 图 2a 表明随 着 $\mathrm{In}^{3+}$ 共掺杂含量的增加, 六方相 $\mathrm{NaYF}_{4}$ 的比例不断提 高, 反之六方相 $\mathrm{NaYF}_{4}$ 的比例不断降低, 说明 $\mathrm{In}^{3+}$ 掺杂 能够诱导 $\mathrm{NaYF}_{4}$ 立方相向六方相转变. 用场发射扫描电

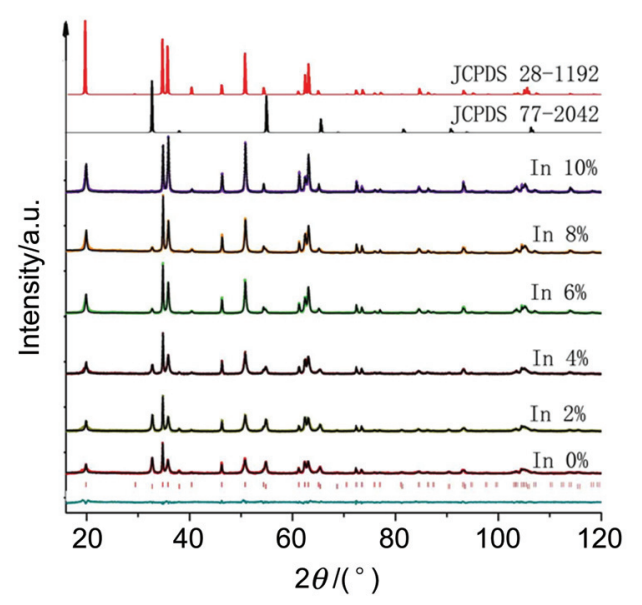

图 1 样品 $\mathrm{A} 0, \mathrm{~A} 1, \mathrm{~A} 2, \mathrm{~A} 3, \mathrm{~A} 4, \mathrm{~A} 5$ 的 X 射线衍射图谱(彩线与黑线分 别是测量与计算得到的图谱), 六方 $\mathrm{NaYF}_{4}$ (JCPDS 28-1192)与立方 $\mathrm{NaYF}_{4}$ (JCPDS 77-2042)标准图谱, In 0\%样品测量与计算的差异谱线 (青色), 以及六方相与立方相的所有衍射峰(红色坚线)

Figure 1 Measured and Rietveld calculated XRD patterns of Sample A0, A1, A2, A3, A4 and A5 (color and black lines indicate measured and calculated pattern, respectively), hexagonal $\mathrm{NaYF}_{4}$ (JCPDS 28-1192) and cubic $\mathrm{NaYF}_{4}$ (JCPDS 77-2042) patterns. Dark cyan line is the difference between measured and calculated pattern of In $0 \%$. And red vertical lines indicate hexagonal and cubic $\mathrm{NaYF}_{4}$ peaks position
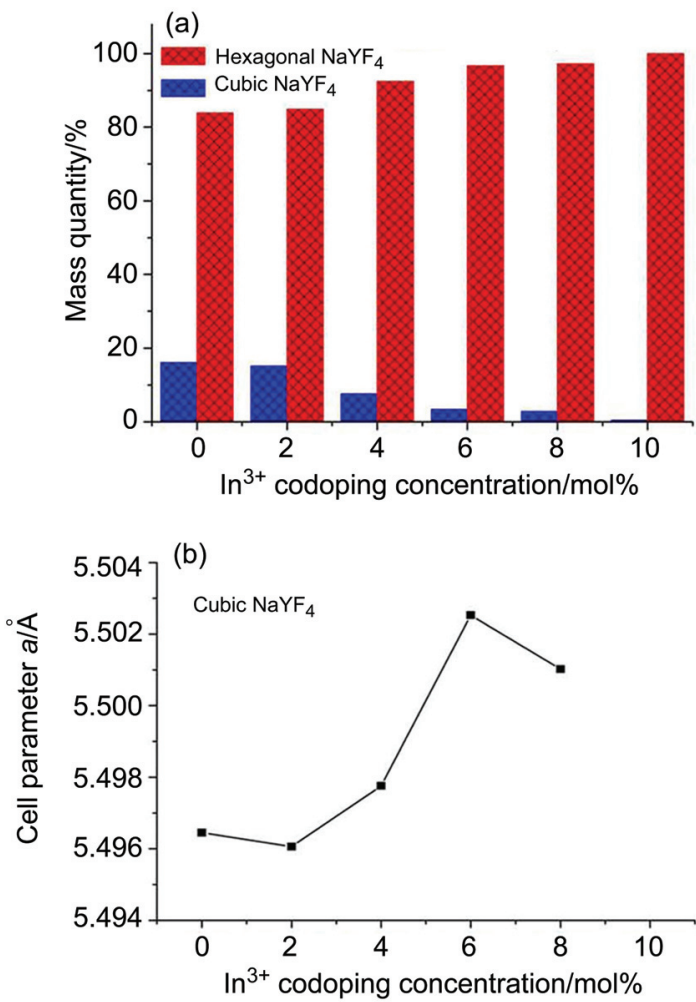

图 2 不同 $\mathrm{In}^{3+}$ 掺杂含量下, (a) 六方 $\mathrm{NaYF}_{4}$ 与立方 $\mathrm{NaYF}_{4}$ 的质量百分 含量, (b) 立方 $\mathrm{NaYF}_{4}$ 晶胞参数 $a$

Figure 2 (a) Mass quantity percentage of cubic and hexagonal $\mathrm{NaYF}_{4}$ with different $\mathrm{In}^{3+}$ concentration codoping. (b) Cell parameter $a$ of cubic $\mathrm{NaYF}_{4}$ with different $\mathrm{In}^{3+}$ concentration codoping

镜(FESEM)进行样品形貌分析结果如图 3 所示, 由纳米 颗粒与微米六方柱组成, 随着 $\mathrm{In}^{3+}$ 含量的增加, 纳米颗 

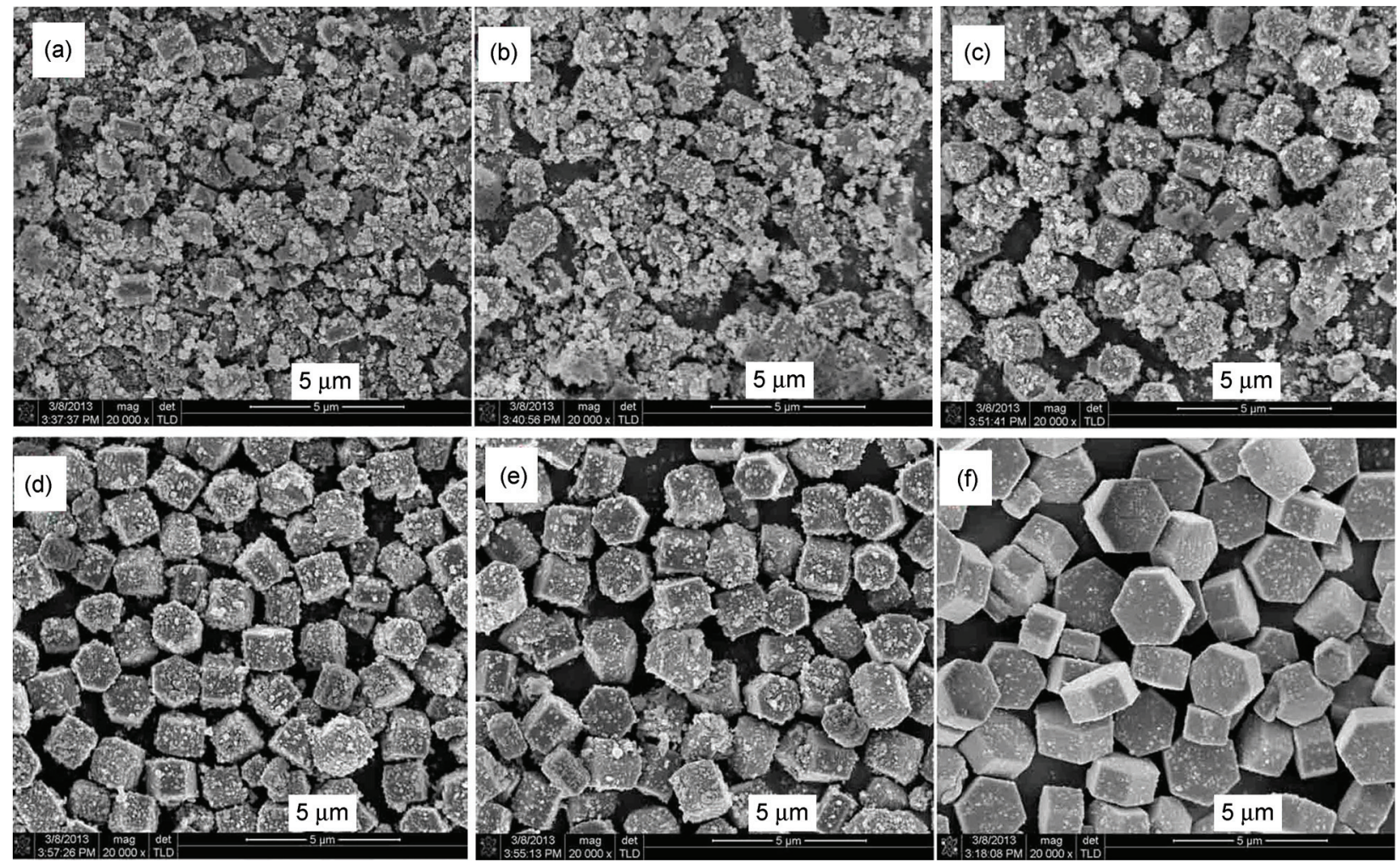

图 3 样品 A0 (a), A1 (b), A2 (c), A3 (d), A4 (e), A5 (f)的场发射扫描电镜照片

Figure 3 FESEM picture of sample A0 (a), A1 (b), A2 (c), A3 (d), A4 (e) and A5 (f), respectively

粒逐渐减少. 微米六方柱显然是六方相 $\mathrm{NaYF}_{4}$, 再对照 XRD 结果中的两相比例变化趋势可知, 纳米尺度的颗 粒主要为立方相 $\mathrm{NaYF}_{4}$.

$\operatorname{In}^{3+}$ 掺杂是如何诱导相转变的呢? 图 2(b)显示随着 $\mathrm{In}^{3+}$ 掺杂含量的增加, 立方 $\mathrm{NaYF}_{4}$ 的晶胞参数 $a$ 先减小, 再增大并于掺杂含量为 $6 \mathrm{~mol} \%$ 时达到最大值, 之后再 次减小. 而图 4(a), 图 4(b)显示随着 $\operatorname{In}^{3+}$ 掺杂含量的增 加, 六方 $\mathrm{NaYF}_{4}$ 的晶胞参数 $a, c$ 与晶胞体积不断减小. 这是由于在六方 $\mathrm{NaYF}_{4}$ 中离子半径较小的 $\mathrm{In}^{3+}$ 代替较大 的 $\mathrm{Y}^{3+}$ 而占据 $\mathrm{Y}^{3+}$ 的位置, 随着 $\mathrm{In}^{3+}$ 掺杂含量的增加, 被 取代的 $\mathrm{Y}^{3+}$ 越来越多, 使得相邻三价离子间距 $R$ 不断减 小[如图 4(c), 图 4(d)] 以至于晶胞体积也不断减小. 立方 $\mathrm{NaYF}_{4}$ 的晶胞参数变化规律为什么会与六方相不同呢? 我们通过透射电镜来寻找答案. 图 5 为样品 $\mathrm{A} 0 \sim \mathrm{A} 5$ 中 $\mathrm{NaYF}_{4}$ 纳米颗粒的高分辨透射电镜 (HRTEM) 照片 (其相 应的 TEM 照片见支持信息). 图 5 中(a1) (f1)为 HRTEM 照片中相应区域经傅里叶变换得到的电子衍射 斑点. 图 5(a1) (A0)显示衍射斑点对应于两种不同的晶 面间距 $(d)$ 为 $0.2741,0.1961 \mathrm{~nm}$, 可分别标定为立方 $\mathrm{NaYF}_{4}$ 的(200)与(220)晶面. 图 5(b1)(A1)显示衍射斑点 对应于三种晶面间距 $(d)$ 为 $0.2723,0.3078,0.1636 \mathrm{~nm}$, 可分别标定为立方 $\mathrm{NaYF}_{4}$ 的(200), (111)与(311) 晶面. 值 得注意的是图 5(b1) (200)晶面的 $d$ 值小于图 5(a1)中的同 一晶面, 这与 XRD 中 $a$ 的变化一致, 可以归因于较小的 $\mathrm{In}^{3+}$ 取代了较大 $\mathrm{Y}^{3+}$. 图 5(c1) (A2)显示衍射斑点对应于
5 种晶面间距 $(d)$ 为 $0.2793,0.1917,0.3118,0.3054,0.1526$ $\mathrm{nm}$. 前三者可分别标定为立方 $\mathrm{NaYF}_{4}$ 的(200), (220)与 (111)晶面, 与 XRD 结果相一致, (220)的 $d$ 值比图 5(a1) 中样品 A0 的同一晶面更大. 后两者无法标定为立方或 六方相 $\mathrm{NaYF}_{4}$ 的任何晶面, 且 $0.3054,0.1526 \mathrm{~nm}$ 分别比 立方 $\mathrm{NaYF}_{4}$ 的(111)与(222)晶面的 $d$ 值小, 从 HRTEM 照 片中相应的区域可见晶格条纹发生了明显的扭曲，表明 这两个衍射点可以归因于晶格的扭曲. 图 5(d1) (A3)显 示衍射斑点对应于 5 种晶面间距 $(d)$ 为 $0.2731,0.3151$, $0.2120,0.2942,0.2831 \mathrm{~nm}$. 其中前两者可分别标定为立 方 $\mathrm{NaYF}_{4}$ 的(200)与(111)晶面; $0.2120,0.2942 \mathrm{~nm}$ 可分别 标定为六方 $\mathrm{NaYF}_{4}$ 的(201)与(110)晶面; $0.2831 \mathrm{~nm}$ 无法 标定为立方或六方相 $\mathrm{NaYF}_{4}$ 的任何晶面. $0.2831 \mathrm{~nm}$ 可 以归因于立方 $\mathrm{NaYF}_{4}$ (200)晶面晶格的扭曲, 后者由立 方相向六方相转变的过渡晶面. 图 5(e1)与图 5(f1)中所 有衍射斑点都可以标定为六方相 $\mathrm{NaYF}_{4}$ 的晶面, 并且在 图 5(e)与图 5(f)中相应的晶格条纹都很规则. 从以上分 析中, 可以得出随着 $\operatorname{In}^{3+}$ 掺杂含量的增加, 因为 $\mathrm{In}^{3+}$ 取 代 $\mathrm{Y}^{3+}$ 的位置, 立方的 $\mathrm{NaYF}_{4}$ 晶格畸变不断增大并发生 向六方相的转变, 使得晶胞参数在转变中间阶段随 $\mathrm{In}^{3+}$ 含量而增大. 按照固溶体理论 ${ }^{[33]}$, 晶格畸变有利于离子 的迁移并将促进相转变的发生, 因此六方 $\mathrm{NaYF}_{4}$ 的质量 分数在 $\mathrm{In}^{3+}$ 掺杂含量在 $4 \mathrm{~mol} \%$ 以上时随着 $\mathrm{In}^{3+}$ 掺杂含 量的增加而明显增大. 而在 $2 \mathrm{~mol} \% \mathrm{In}^{3+}$ 掺杂时, 由于晶 格扭曲较小, 六方 $\mathrm{NaYF}_{4}$ 的质量分数没有明显的变化. 

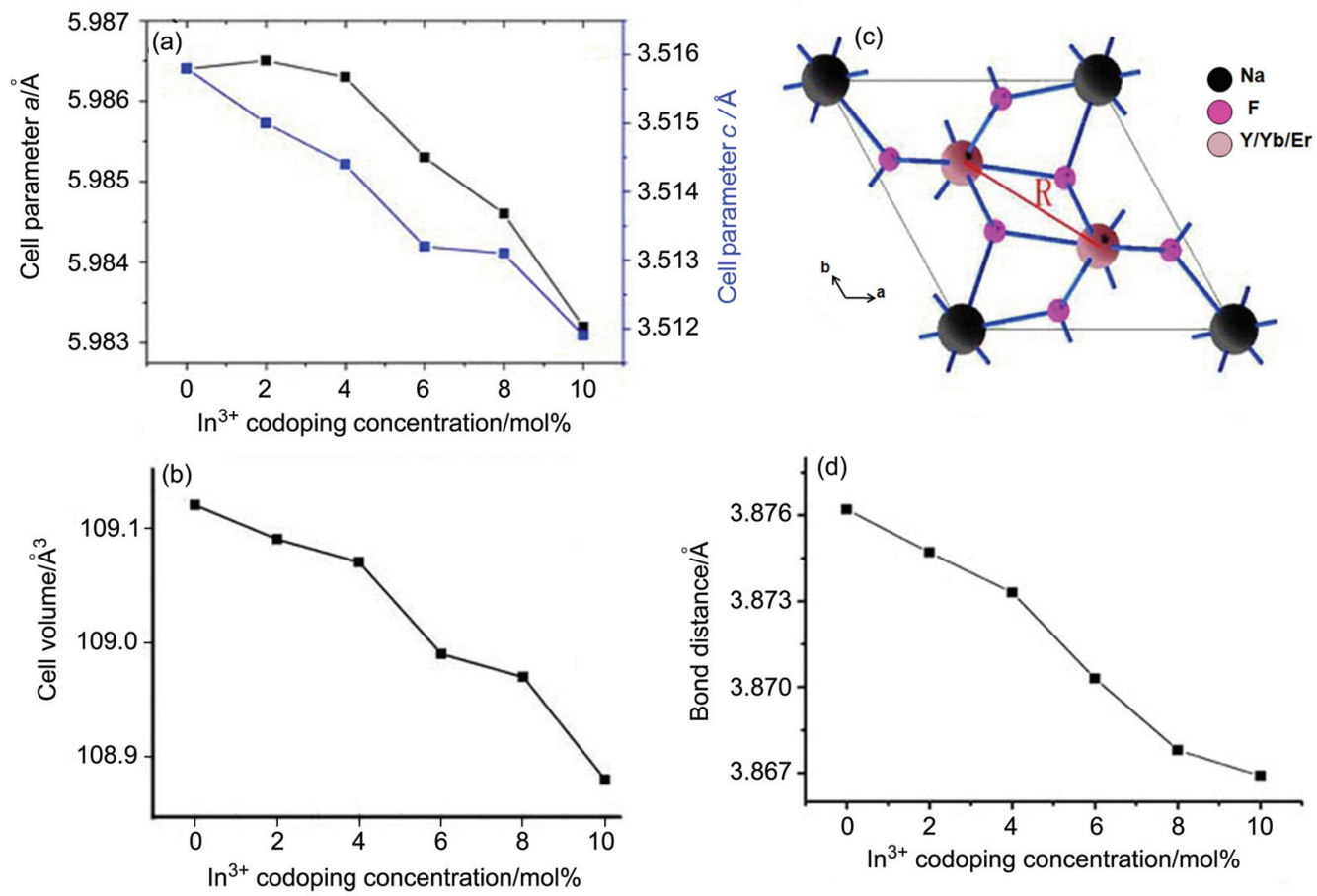

图 4 六方相 $\mathrm{NaYF}_{4}$ 晶体结构随 $\mathrm{In}^{3+}$ 掺杂含量的变化: (a) 晶胞参数 $a, c$; (b) 晶胞体积; (c) 晶胞示意图; (d)相邻三价离子间距 $(\mathrm{c})$ 中的 $(R)$

Figure 4 (a) cell parameter $a, c$ and (b) cell volume of hexagonal $\mathrm{NaYF}_{4}$. (c) hexagonal $\mathrm{NaYF}_{4}$ crystal structure sketch, (d) the bond distance of two interfacing trivalent ions described as " $R$ " in (c) changed with different $\operatorname{In}^{3+}$ codoping concentration
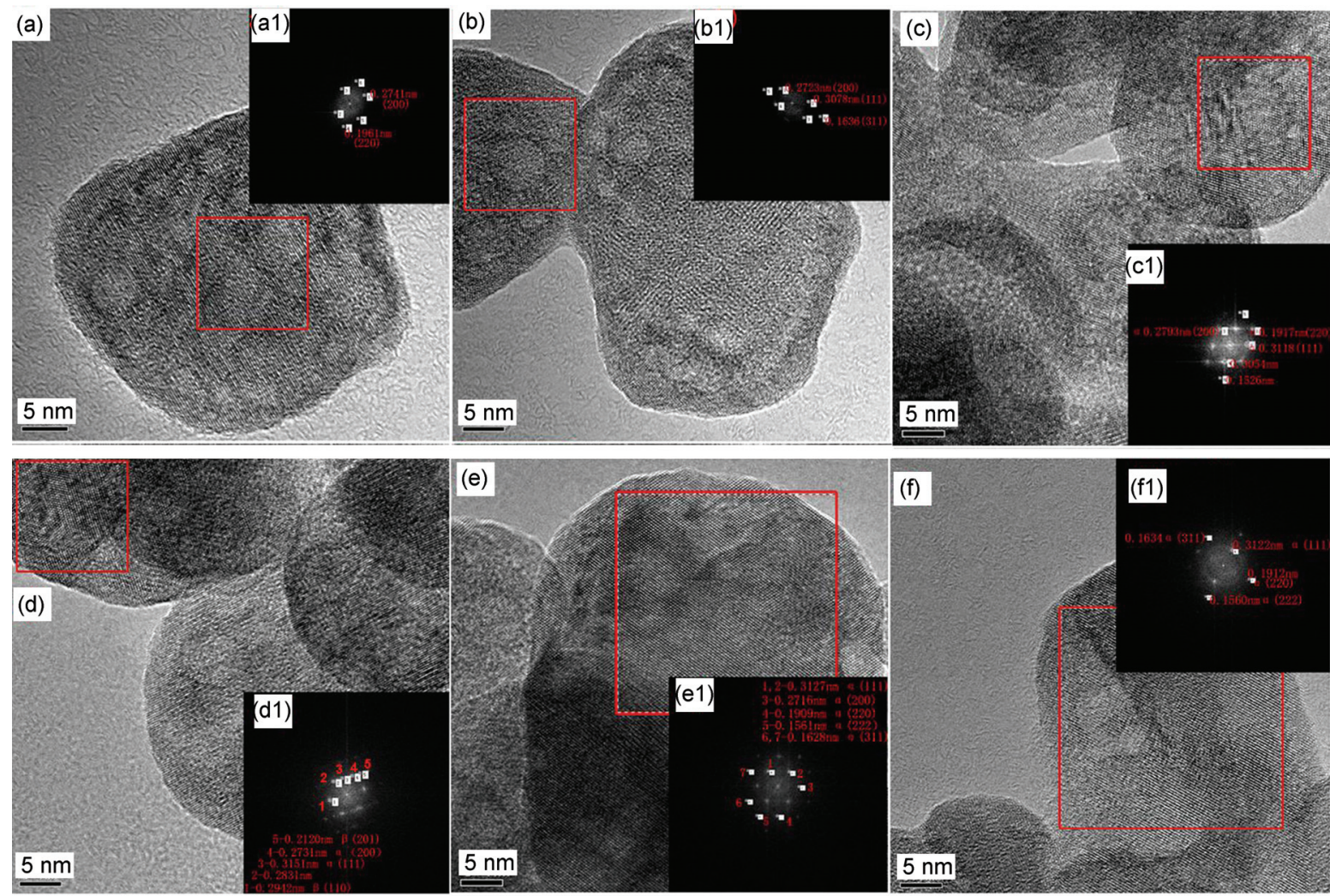

图 5 A0 (a), A1 (b), A2 (c), A3 (d), A4 (e), A5 (f)的高分辨透射电镜照片; $\left(\mathrm{a}_{1}\right),\left(\mathrm{b}_{1}\right),\left(\mathrm{c}_{1}\right),\left(\mathrm{d}_{1}\right),\left(\mathrm{e}_{1}\right),\left(\mathrm{f}_{1}\right)$ 相应的电镜照片中红框区域由傅里叶变换得到 的电子衍射斑点

Figure 5 HR-TEM pictures of A0 (a), A1 (b), A2 (c), A3 (d), A4 (e) and A5 (f), and ( $\left.a_{1}\right),\left(b_{1}\right),\left(c_{1}\right),\left(d_{1}\right),\left(e_{1}\right)$ and $\left(f_{1}\right)$ the FFT patterns of the corresponding HR-TEM red square areas, respectively 
图 2 中晶胞参数与两相质量分数的变化规律与图 5 中晶 格的扭曲与变化趋势相一致, 都随着 $\mathrm{In}^{3+}$ 不同掺杂含量 而变化.

图 6 为样品 $\mathrm{A} 0 \sim \mathrm{A} 5$ 中六方相 $\mathrm{NaYF}_{4}$ 的 TEM 照片, 六方柱的晶粒尺寸与底面直径和柱高的径长比都随着 $\mathrm{In}^{3+}$ 掺杂含量的增加而增大. 径长比的增大表明 $\mathrm{In}^{3+}$ 掺 杂能够促进(001)晶面的生长. 立方相向六方相的转变 也是一个溶解与重结晶的过程. 由于六方相 $\mathrm{NaYF}_{4}$ (110), (101)与(011)面的三价离子密度高于 $(001)$ 面, 当 越来越多的 $\mathrm{In}^{3+}$ 随着掺杂含量的增加取代 $\mathrm{Y}^{3+}$ 并占据 $\mathrm{Y}^{3+}$ 的位置时, (110), (101)与(011)面的晶格畸变与表面 能增大程度都要超过(001)面, 因而相对促进了 [110], [101] 与 [011]方向的生长, 导致了径长比的增大.

\section{$2.2 \mathrm{In}^{3+}$ 共掺杂对六方 $\mathrm{NaYF}_{4}$ 上转换发光性能的影响}

$\mathrm{NaY}_{0.92} \mathrm{Yb}_{0.03} \mathrm{Er}_{0.02} \mathrm{~F}_{4}$ 与 $0,1,2,3,4,5 \mathrm{~mol} \% \mathrm{In}^{3+}$ 共掺 杂, 在 $190{ }^{\circ} \mathrm{C}$ 烘箱中恒温反应 $12 \mathrm{~h}$ 得到的样品分别标记 为 B0, B1, B2, B3, B4, B5, 用于上转换发光性能的研究. $\mathrm{NaYF}_{4}$ 的六方相相对于立方相是一个热力学稳定的相, 提高反应温度，延长反应时间能够促进六方相 $\mathrm{NaYF}_{4}$ 的 形成 ${ }^{[18]}$. 样品 $\mathrm{B} 0 \sim \mathrm{B} 5$ 的三价阳离子的元素定量分析如 支持信息表 2 所示, 元素分析结果与实验设计基本相符. XRD 谱如图 7 所示, 所有的衍射谱都能够标识为六方相
$\mathrm{NaYF}_{4}$ (JCPDS 卡片号 28-1192)，且没有其它未标明的 衍射峰，表明都是纯相的六方 $\mathrm{NaYF}_{4}$. 与 $\mathrm{A} 0 \sim \mathrm{A} 5$ 同样 进行 Rietveld 精修, 所有的精修结果各评价 $R$ 因子都小 于 6 , 计算图谱与实测图谱吻合的很好, 如图 7 所示. 晶 胞参数则见于图 8 , 所有的参数都随 $\mathrm{In}^{3+}$ 共掺杂含量的 增加而减小, 说明 $\mathrm{In}^{3+}$ 掺入晶格中并占据了 $\mathrm{Y}^{3+}$ 的位置.

B0 B5 的上转换发光性能通过图 9 中的上转换发 射光谱和衰减曲线来考察. 图 9(a)中的荧光发射峰可分 别归属为以下跃迁: ${ }^{2} \mathrm{H}_{11 / 2} \rightarrow{ }^{4} \mathrm{I}_{15 / 2}(\approx 520 \mathrm{~nm}),{ }^{4} \mathrm{~S}_{3 / 2} \rightarrow{ }^{4} \mathrm{I}_{15 / 2}$ $(\approx 544 \mathrm{~nm})$ 和 ${ }^{4} \mathrm{~F}_{9 / 2} \rightarrow{ }^{4} \mathrm{I}_{15 / 2}(\approx 654 \mathrm{~nm})$. 这些峰的上转换 发射强度全都随着 $\mathrm{In}^{3+}$ 共掺杂含量的增加而增大, 并且 在 $\mathrm{In}^{3+}$ 掺杂含量为 $3 \mathrm{~mol} \%$ 时达到最大值, 此后随 $\mathrm{In}^{3+}$ 共 掺杂含量进一步增加而减小. $520,544,654 \mathrm{~nm}$ 的上转换 发射衰减曲线可以用单指数衰减函数进行拟合，求出相 应的荧光寿命常数, 寿命分析结果分别如图 $9\left(b_{1}\right)$, 图 $9\left(c_{1}\right)$, 图 $9\left(d_{1}\right)$ 所示. 从中可以看出, 不同发射峰的苂光 衰减时间常数都随着 $\mathrm{In}^{3+}$ 共掺杂含量的增加而减小, 654 $\mathrm{nm}$ 发射峰的苂光衰减时间常数比 $520 \mathrm{~nm}$ 和 $544 \mathrm{~nm}$ 的 要长. 由于 $\mathrm{In}^{3+}$ 共掺杂含量的不同引起的六方 $\mathrm{NaYF}_{4}$ 上 转换发光特性的变化可以归因于如下三个原因. 其一, 六方相 $\mathrm{NaY}_{0.95-\mathrm{x}} \mathrm{Yb}_{0.03} \mathrm{Er}_{0.02} \mathrm{~F}_{4}$ 中 $\mathrm{Yb}^{3+}, \mathrm{Er}^{3+}$ 在 $\mathrm{NaYF}_{4}$ 基质 中掺杂并取代 $\mathrm{Y}^{3+}(90 \mathrm{pm})$ 而占据 $\mathrm{Y}^{3+}$ 的位置. 当 $\mathrm{In}^{3+}$ 共
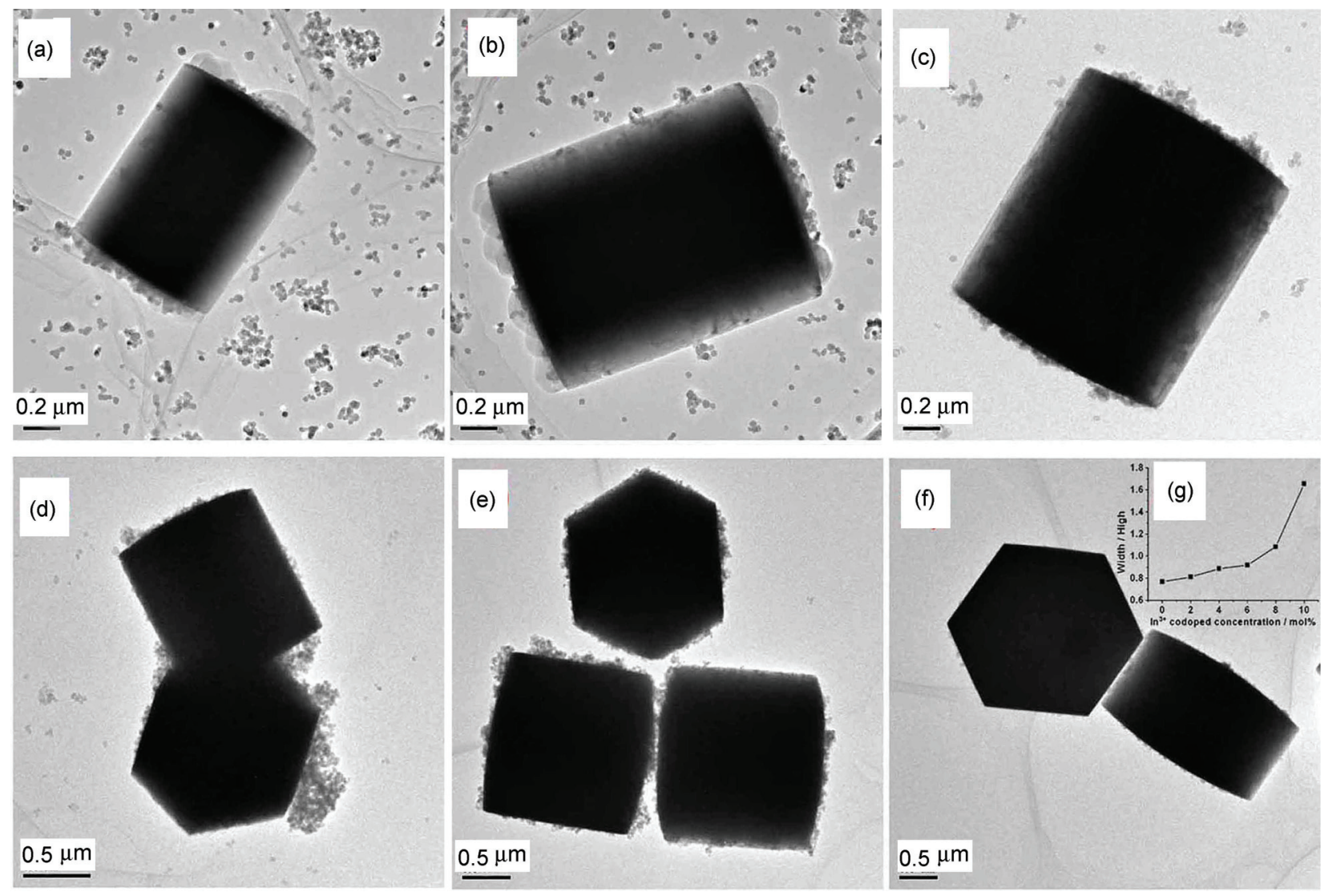

图 6 样品 $\mathrm{A} 0$ (a), A1 (b), A2 (c), A3 (d), A4 (e), A5 (f)的 TEM 照片; (g) 径长比随 $\mathrm{In}^{3+}$ 掺杂含量的变化趋势

Figure 6 TEM pictures focusing on the hexagonal $\mathrm{NaYF}_{4}$ of sample A0 (a), A1 (b), A2 (c), A3 (d), A4 (e) and A5 (f), respectively. (g) the changing trend of the ratio of width to thickness with rising of the $\mathrm{In}^{3+}$ codoping concentration 


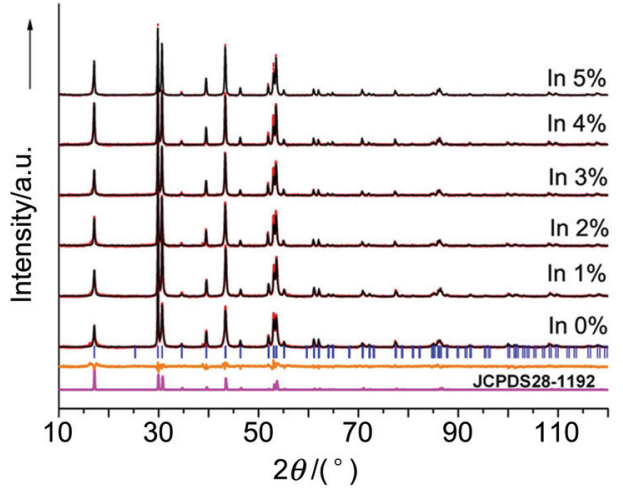

图 7 样品 B0, B1, B2, B3, B4, B5 的 X 射线衍射图谱(红线与黑线分 别是测量与计算得到的图谱), In $0 \%$ 样品测量与计算的差异谱线(黄 色), 六方相 $\mathrm{NaYF}_{4}$ 的所有衍射峰(蓝色坚线), 以及六方 $\mathrm{NaYF}_{4}$ (JCPDS 28-1192)标准谱线(粉色).

Figure 7 Measured and Rietveld calculated XRD pattern of B0, B1, B2, B3, B4, B5. Red and black lines show measured and calculated patterns, respectively. Yellow line is the difference between measured and calculated patterns of In $0 \%$. Blue vertical lines indicate hexagonal $\mathrm{NaYF}_{4}$ peak position. Pink line is the XRD pattern of JCPDS 28-1192

掺杂进入六方 $\mathrm{NaYF}_{4}$ 中后, 它也同样要占据 $\mathrm{Y}^{3+} / \mathrm{Yb}^{3+} /$ $\mathrm{Er}^{3+}$ 的位置. 更小的 $\operatorname{In}^{3+}(80 \mathrm{pm})$ 对晶格的掺入会改变 $\mathrm{Yb}^{3+}$ 和 $\mathrm{Er}^{3+}$ 周围晶体结构的对称性, 从而使晶格发生畸 变 $\mathrm{Yb}^{3+}$ 和 $\mathrm{Er}^{3+}$ 周围晶体场产生不对称性. 这些变化还 会随着 $\mathrm{In}^{3+}$ 共掺杂含量的增加而加剧. 按照 Judd-Ofelt 理论 ${ }^{[30,31]} ， \Omega$ 参数对环境十分敏感, 能够为稀土离子周 围晶体场对称性的变化而改变. 而非对称的晶体场环境 会导致 $\mathrm{Yb}^{3+}$ 与 $\mathrm{Er}^{3+}$ 发生超灵敏电子跃迁, 继而提高 $\mathrm{Er}^{3+}$ 激发态的布居数, 从而增强上转换发光. 其二, 当 $\mathrm{In}^{3+}$ 共掺杂含量进一步增大时, 分散性减小而聚集成簇, 使 得稀土离子周围晶体场的不对称性反而减小 ${ }^{[29]}$, 结果 上转换发光强度由于超灵敏电子跃迁的减少而减弱. 从 光谱中可知, $3 \mathrm{~mol} \%$ 为 $\mathrm{In}^{3+}$ 最佳掺杂含量, 此时上转换 发光强度最大. 其三, 荧光寿命随 $\mathrm{In}^{3+}$ 共掺杂含量的增 加而减小, 可以归因于晶格畸变的加剧. 晶格畸变会诱 导电子从激发态驰豫到基态, 从而减少交叉弛豫上转换
的可能性使 $\mathrm{Yb}^{3+}$ 的弛豫淬灭. $\mathrm{Er}^{3+}$ 的 ${ }^{2} \mathrm{H}_{11 / 2},{ }^{4} \mathrm{~S}_{3 / 2}$ 和 ${ }^{4} \mathrm{~F}_{9 / 2}$ 激发态的布居数绝大多数来自于基态吸收与协同上转 换, 它们的苂光寿命也因此随着 $\mathrm{In}^{3+}$ 共掺杂含量的增加 而减小. 由以上分析可知晶体场的非对称性与晶格畸变 是一体两面, 为获得最佳的上转换发光性能要同时加以 考虑.

\section{3 结论}

综上所述, 我们引入 $\mathrm{In}^{3+}$ 与 $\mathrm{Yb}^{3+} / \mathrm{Er}^{3+}$ 共掺杂 $\mathrm{NaYF}_{4}$, 对 $\mathrm{In}^{3+}$ 对 $\mathrm{NaYF}_{4}$ 相转变与上转换发光特性的影 响进行了研究. 研究表明, $\mathrm{In}^{3+}$ 的引入能够促使晶格扭 曲, 从而促进 $\mathrm{NaYF}_{4}$ 由立方相向六方相转变, 是降低反 应温度与时间的有效途径; $\mathrm{In}^{3+}$ 的共掺杂还能使六方相 $\mathrm{NaYF}_{4}$ 的上转换发光增强并在 $3 \mathrm{~mol} \% \mathrm{In}^{3+}$ 掺杂时达到 峰值, 同时苂光寿命由于 $\mathrm{In}^{3+}$ 共掺杂造成的晶格畸变而 减小.

\section{4 实验部分}

\section{1 制备}

$\mathrm{NaY}_{0.92} \mathrm{Yb}_{0.03} \mathrm{Er}_{0.02} \mathrm{~F}_{4}$ 与 $0,2,4,6,8,10 \mathrm{~mol} \% \mathrm{In}^{3+}$ 共 掺杂的样品分别标记为 $\mathrm{A} 0, \mathrm{~A} 1, \mathrm{~A} 2, \mathrm{~A} 3, \mathrm{~A} 4, \mathrm{~A} 5$, 用于相 转变的研究. 典型的合成工程如下, 将上述硝酸盐以特 定的比例加入含有 $0.4 \mathrm{mmol}$ EDTA 二钠与 $10 \mathrm{~mL}$ 油酸 的 $20 \mathrm{~mL}$ 水溶液中. 待剧烈搅拌 $30 \mathrm{~min}$ 后，向溶液中逐 滴加入含有 $0.2 \mathrm{mmol} \mathrm{NaF}$ 和 $0.2 \mathrm{mmol} \mathrm{NH}_{4} \mathrm{HF}_{2}$ 的 $25 \mathrm{~mL}$ 乙醇溶液, 并加入 $1 \mathrm{~mol} / \mathrm{L} \mathrm{HF}$ 溶液将 $\mathrm{pH}$ 调至 3.0. 继续 摚拌 $30 \mathrm{~min}$ 后, 将混合物移入聚四氟乙烯反应釜, 在 $170{ }^{\circ} \mathrm{C}$ 烘箱中恒温反应 $8 \mathrm{~h}$. 最后将所得样品用环已烷、 乙醇、水分别洗 3 次, 在 $60{ }^{\circ} \mathrm{C}$ 烘箱中干燥. 另一组样品 用 $0,1,2,3,4,5 \mathrm{~mol} \% \mathrm{In}^{3+}$ 共掺杂, 在 $190{ }^{\circ} \mathrm{C}$ 烘箱中恒温 反应 $12 \mathrm{~h}$, 其它条件不变, 以获得纯六方相的 $\mathrm{NaYF}_{4}$. 这组样品依次标记为 B0, B1, B2, B3, B4, B5, 用于上转 换发光性能的研究.

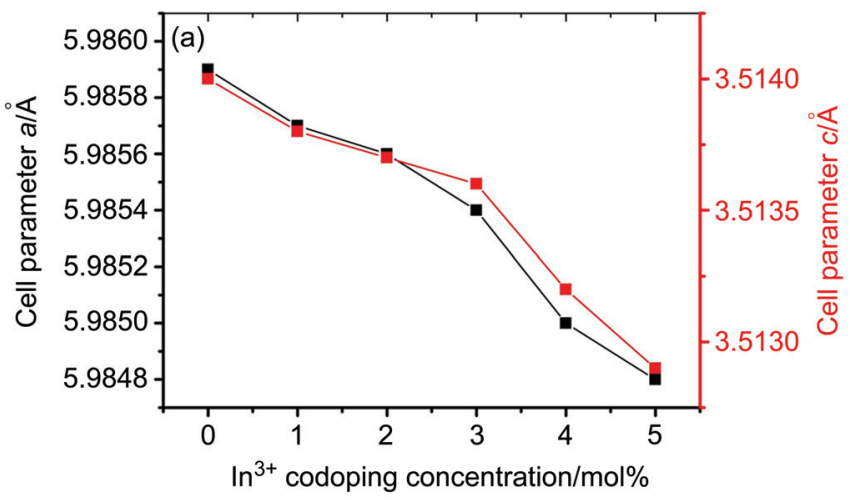

图 8 样品 B0, B1, B2, B3, B4, B5 的(a)晶胞参数 $a, c$ 与(b)晶胞体积 $(V)$

Figure 8 (a) and (b) cell parameter $a, c$ and $V$ of sample B0, B1, B2, B3, B4 and B5, respectively 

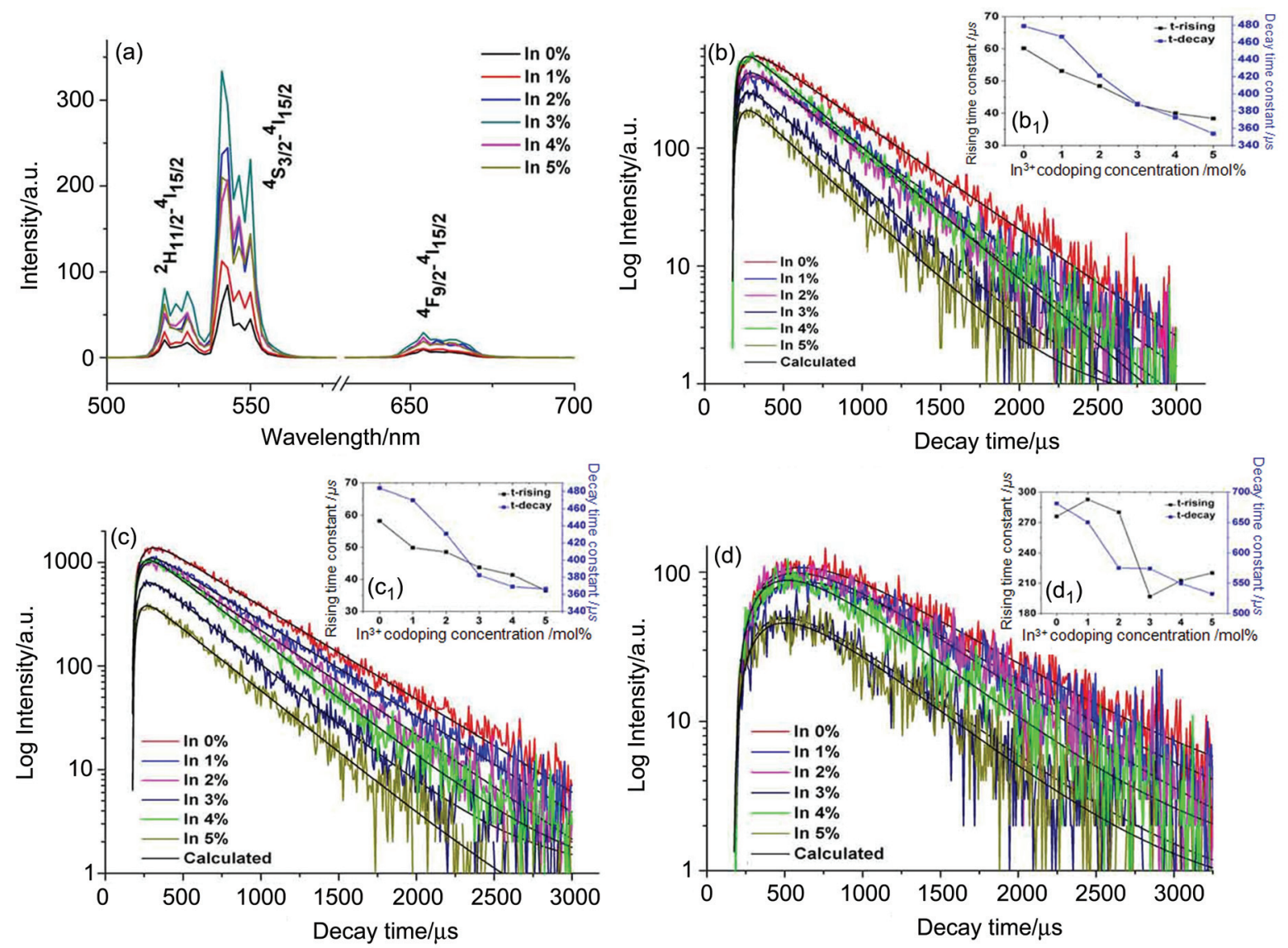

图 9 B0, B1, B2, B3, B4, B5 的光谱数据: (a)发射谱, (b) $520 \mathrm{~nm}$, (c) $544 \mathrm{~nm}$, (d) $654 \mathrm{~nm}$ 的菼光衰减曲线, (b1) $520 \mathrm{~nm}$, (c1) $544 \mathrm{~nm},(\mathrm{~d} 1) 654 \mathrm{~nm}$ 的苂 光寿命的变化趋势(黑色曲线为上升时间常数变化趋势, 蓝色曲线为衰减时间常数变化趋势)

Figure 9 (a) Emission spectra and (b) $520 \mathrm{~nm}$, (c) $544 \mathrm{~nm}$, (d) $654 \mathrm{~nm}$ emission decay curves of sample B0, B1, B2, B3, B4 and B5, respectively. decay time and rising time constant changing trends of $\left(b_{1}\right) 520 \mathrm{~nm},\left(c_{1}\right) 544 \mathrm{~nm}$ and $\left(\mathrm{d}_{1}\right) 654 \mathrm{~nm}$ emissions, respectively (the black curves are changing trends of the rising time constants, while the blue curves are those of the decay time constants)

\section{2 表征}

$X$ 射线多晶衍射(XRD)图谱用帕纳科公司的 X'pert ProMPD 仪器收录, 测试条件: Co 靶, 波长为 0.178901 $\mathrm{nm}$, 扫描速度 $1\left(^{\circ}\right) / \mathrm{min}, 2 \theta$ 扫描范围 $15^{\circ} \sim 120^{\circ}$. 场发射 扫描电镜(FESEM)照片是在 FEI Nova NanaSEM230 扫 描电子显微镜上拍摄的. 高分辨透射电镜(HRTEM) 照 片是在 FEI Tecnai G2 F20 S-TWIN 场发射透射电镜上 拍摄的, 工作电压为 $200 \mathrm{kV}$. 上转换发光光谱和时间衰 减谱是在 Edinburgh FSLP920 仪器上测试的, 激发源波 长为 $980 \mathrm{~nm}$, 泵浦功率为 $5 \mathrm{~W} / \mathrm{cm}^{2}$, 激光脉冲宽度 $5 \mathrm{ns,}$ 频率 $10 \mathrm{~Hz}$, 使用积分球. 所有样品的测试严格以同一 条件在室温下进行.

\section{References}

[1] Wang, F.; Liu, X. G. Chem. Soc. Rev. 2009, 38, 976.

[2] Heer, S.; Kompe, K.; Gudel, H. U.; Haase, M. Adv. Mater. 2004, 16, 2102.

[3] Vennerberg, D.; Lin, Z. Q. Sci. Adv. Mater. 2011, 3, 26.

[4] Chen, W. J. Nanosci. Nanotechnol. 2008, 8, 1019.

[5] Wang, M.; Mi, C. C.; Zhang, Y. X.; Liu, J. L.; Li, F.; Mao, C. B.; Xu, S. K. J. Phys. Chem. C 2009, 113, 19021.
[6] Ryu, J.; Park, H. Y.; Kim, K.; Kim, H.; Yoo, J. H.; Kang, M.; Im, K.; Grailhe, R.; Song, R. J. Phys. Chem. C 2010, 114, 21077.

[7] Nagarajan, S.; Zhang, Y. Nanotechnology 2011, 22, 395101.

[8] Chen, F.; Bu, W. B.; Zhang, S. J.; Liu, J. N.; Fan, W. P.; Zhou, L. P.; Peng, W. J.; Shi, J. L. Adv. Funct. Mater. 2013, 23, 298.

[9] Righini, G. C.; Ferrari, M. Riv. Nuovo. Cimento. 2005, $28,1$.

[10] Zhou, S. S.; Deng, K. M.; Wei, X. T.; Jiang, G. C.; Duan, C. K.; Chen, Y. H.; Yin, M. Opt. Commun. 2013, 291, 138.

[11] Vetrone, F.; Naccache, R.; Zamarron, A.; de la Fuente, A. J.; Sanz-Rodriguez, F.; Maestro, L. M.; Rodriguez, E. M.; Jaque, D.; Sole, J. G.; Capobianco, J. A. ACS Nano 2010, 4, 3254.

[12] Li, T. G.; Liu, S. W.; Zhang, H. P.; Wang, E. H.; Song, L. J.; Wang, P. J. Mater. Sci. 2011, 46, 2882

[13] van der Ende, B. M.; Aarts, L.; Meijerink, A. Phys. Chem. Chem. Phys. 2009, 11, 11081.

[14] Wang, F.; Deng, R. R.; Wang, J.; Wang, Q. X.; Han, Y.; Zhu, H. M.; Chen, X. Y.; Liu, X. G. Nat. Mater. 2011, 10, 968.

[15] Li, Z. Q.; Li, X. D.; Liu, Q. Q.; Chen, X. H.; Sun, Z.; Liu, C.; Ye, X. J.; Huang, S. M. Nanotechnology 2012, 23, 345606.

[16] Zhang, J.; Shen, H. O.; Guo, W.; Wang, S. H.; Zhu, C. T.; Xue, F.; Hou, J. F.; Su, H. Q.; Yuan, Z. B. J. Power Sources 2013, 226, 47.

[17] Suyver, J. F.; Aebischer, A.; Biner, D.; Gerner, P.; Grimm, J.; Heer, S.; Kramer, K. W.; Reinhard, C.; Gudel, H. U. Opt. Mater. 2005, 27, 1111 .

[18] Liang, X.; Wang, X.; Zhuang, J.; Peng, Q.; Li, Y. D. Adv. Funct. Mater. 2007, 17, 2757.

[19] Wang, L. Y.; Li, P.; Li, Y. D. Adv. Mater. 2007, 19, 3304.

[20] Mai, H. X.; Zhang, Y. W.; Si, R.; Yan, Z. G.; Sun, L. D.; You, L. P.; Yan, C. H. J. Am. Chem. Soc. 2006, 128, 6426.

[21] Mai, H. X.; Zhang, Y. W.; Sun, L. D.; Yan, C. H. J. Phys. Chem. C 2007, 111, 13721 
[22] Mai, H. X.; Zhang, Y. W.; Sun, L. D.; Yan, C. H. J. Phys. Chem. C 2007, 111, 13730.

[23] Li, C. X.; Quan, Z. W.; Yang, P. P.; Yang, J.; Lian, H. Z.; Lin, J. J. Mater. Chem. 2008, 18, 1353.

[24] Huang, Q. M.; Yu, H.; Zhang, X. Q.; Yu, J. C. Acta Chim. Sinica 2013, 71, 1071 (黄清明, 俞瀚, 张新奇, 俞建长, 化学学报, 2013, $71,1071)$.

[25] Chen, J.; Guo, C. R.; Wang, M.; Huang, L.; Wang, L. P.; Mi, C. C.; Li, J.; Fang, X. X.; Mao, C. B.; Xu, S. K. J. Mater. Chem. 2011, 21, 2632.
[26] Wang, F.; Liu, X. G. J. Am. Chem. Soc. 2008, 130, 5642.

[27] Auzel, F. Chem. Rev. 2004, 104, 139.

[28] Chen, D. Q.; Yu, Y. L.; Huang, F.; Huang, P.; Yang, A. P.; Wang, Y. S. J. Am. Chem. Soc. 2010, 132, 9976.

[29] Huang, Q.; Yu, J.; Ma, E.; Lin, K. J. Phys. Chem. C 2010, 114, 4719.

[30] Judd, B. R. Phys. Rev. 1962, 127, 750.

[31] Ofelt, G. S. J. Chem. Phys. 1962, 37, 511.

[32] Dou, Q. Q.; Zhang, Y. Langmuir 2011, 27, 13236.

[33] Lifshitz, I. M.; Slyozov, V. V. J. Phys. Chem. Solids 1961, 19, 35.

(Qin, X.) 